\title{
Systematic Review: Safety of Intravesical Therapy for Bladder Cancer in the Era of COVID-19
}

\author{
Cale E. Leeson ${ }^{1} \cdot$ Asmaa Ismail $^{1} \cdot$ Mohamed M. Hashad $^{2} \cdot$ Hazem Elmansy $^{1} \cdot$ Walid Shahrour $^{1} \cdot$ Owen Prowse $^{1} \cdot$ \\ Ahmed Kotb ${ }^{1}$ (D)
}

Accepted: 13 August 2020 / Published online: 18 August 2020

(C) Springer Nature Switzerland AG 2020

\begin{abstract}
A novel coronavirus has emerged in late 2019 capable of causing a severe respiratory disease known as COVID-19. Its pathogenesis appears to be the initiation of an immune response and resulting cytokine storm that damages the healthy lung tissue of the host. Some epidemiological studies found bacillus Calmette-Guérin (BCG) vaccine can help to decrease morbidity and mortality of the viral infection. We aim to review and summarize what is known about COVID-19 and the current implications of intravesical BCG with regard to the disease.
\end{abstract}

Keywords COVID-19 $\cdot$ Coronavirus $\cdot \mathrm{BCG} \cdot$ Bladder cancer

\section{Introduction}

In December 2019, a novel coronavirus, now officially known as severe acute respiratory syndrome coronavirus 2 (SARS$\mathrm{CoV}-2)$, emerged in China and is capable of causing the infectious disease called coronavirus disease 2019 (COVID-19). [1] Since then, the World Health Organization has declared a global pandemic, with 1,051,635 global cases of COVID-19, resulting in 56,985 deaths as of April 4, 2020. [2] The clinical manifestations of COVID-19 are varied, ranging from mild symptoms such as fever, dyspnea and cough to more severe complications in the case of acute respiratory distress syndrome (ARDS), septic shock and death. Pathogenesis of the disease likely involves initiation of an immune response, resulting in the production of cytokines in a "cytokine storm" that damages healthy lung tissue of the host. [3]

Future clinical trials are being arranged to determine if bacillus Calmette-Guérin (BCG), an attenuated version of

This article is part of the Topical Collection on Covid-19

Ahmed Kotb

drahmedfali@gmail.com

1 Urology Department, Northern Ontario School of Medicine, Thunder Bay, Ontario, Canada

2 Urology Department, Alexandria University, Alexandria, Egypt
Mycobacterium bovis and vaccine against tuberculosis, has the ability to upregulate the immune system against the novel coronavirus. [4] Furthermore, BCG immunotherapy is often utilized in the field of urology for the treatment of bladder cancer. It is not yet determined if intravesical therapy will have a positive or negative effect on the immune system in bladder cancer patients with COVID-19.

Bladder cancer is a common urologic condition, with urothelial carcinoma comprising the largest proportion of cases (90\%). [5] In addition, 75\% of new bladder cancer cases will be non-muscle invasive bladder cancer (NMIBC), typically treated with a high-quality transurethral resection of the bladder tumour (TURBT), followed by either intravesical chemotherapy or immunotherapy. [5-7] Compared with other intravesical therapies, BCG is the only agent associated with a decreased cancer progression risk compared with TURBT alone but is associated with a higher risk of adverse events. [8] The mechanism of action of BCG is still relatively unclear but may involve an upregulation of the immune system characterized by induced expression of cytokines in the urine and bladder tissue. [9] Intravesical BCG treatment in immunocompromised patients remains a relative contraindication in some guidelines ${ }^{7}$, while its role in the treatment of acute immunological disorders remains uncertain.

In this review, we aim to summarize what is known about the immunologic mechanisms of COVID-19, the current recommendations, mechanisms of action and adverse effects of intravesical BCG in bladder cancer and determine if 
intravesical BCG is safe in patients that are suspected or confirmed to have COVID-19. In addition, implications for BCG immunotherapy in the treatment of viruses and immunological disorders will be explored.

\section{Material and Methods}

Our article followed PRISMA checklist. This review article is IRB exempt as we did not include any data from our patients. A comprehensive search of PubMed was performed for available literature on the effect of COVID-19 on immune response, as well as current intravesical management of bladder cancer. We reviewed relevant articles focusing on the mechanism of action of intravesical BCG and chemotherapy, including systemic outcomes and side effects from intravesical treatment, and its safety and efficacy in immunocompromised patients or those with acute immunological disorders. Search terms included "SARS-CoV-2" or "COVID-19" combined with "immune response", "intravesical BCG" combined with "bladder cancer", "urothelial carcinoma", "adverse events", "side effects", "mechanism of action", "immunocompromised" and "immunological disease". References from review articles and guidelines were also evaluated for articles that may have been missed. Standard electronic search methods using PubMed database and Google Scholar were employed. There were no limits set on publication date, though literature within the last decade was prioritized to formulate a narrative review. All searches were performed using standard search techniques with the exclusion of editorials and letters.

\section{Results}

Forty-five references were of relevance to our work. No study did actually look for the direct correlation between COVID-19 and intravesical BCG but were correlating with different diseases that were of relevance. We avoided guidelines inclusion into our manuscript as guidelines during that stressful time were mainly looking at decreasing patients travel to hospital mainly to decrease their exposure to infection rather than for the actual risk benefit ratio of $\mathrm{BCG}$ itself.

\section{Discussion}

SARS-CoV-2 infection has been described in three stages: an asymptomatic incubation period up to 14 days (stage I), a nonsevere symptomatic period with the presence of the virus (stage II) and a severe respiratory symptomatic stage with high viral load (stage III). [3, 10] Stages I and II require a specific adaptive immune response in healthy hosts to eliminate the virus and prevent progression to stage III; thus, strategies to boost immune responses may play a role in the early stages of COVID-19. [10] When this immune response is impaired, the virus will propagate and result in massive destruction of hostaffected tissues. [10] If the disease progresses to stage III, the severe respiratory phase, lymphocytopenia and high levels of pro-inflammatory cytokines have been observed, suggesting a "cytokine storm" plays a major role in the pathogenesis of COVID-19. [3, 10-15].

Interestingly, a review by D'Antiga [16] of the current and past coronavirus outbreaks found that immunosuppressed patients are not at increased risk for more severe pulmonary disease compared with the general population and concluded there is no reason to postpone life-saving treatments such as transplantation or chemotherapy for cancer. This may be related to the implications of the host immune system response leading to tissue damage in healthy patients that advance to the severe stage of COVID-19, whereas those who are immunocompromised may have a weakened response that could be protective.

Therefore, the pathogenesis of COVID-19 appears to be heavily related to a cytokine storm resulting in inflammatory damage of host lung tissue and has the potential to result in ARDS and death, especially in older patients with comorbidities.

\section{Mechanism of Action of Intravesical BCG}

To help guide future implications for intravesical treatment in COVID-19 and immunological disorders, it is crucial to review our understanding of the mechanisms of action for BCG intravesical therapy. As previously discussed, there are two branches of intravesical therapy for the treatment of bladder cancer: chemotherapy and immunotherapy. Overall, immunotherapy acts with the patient's immune system to upregulate and encourage destruction of cancer cells.

In general, our understanding of the mechanisms of action for intravesical immunotherapy with BCG is still unclear. It is thought that the instillation of intravesical BCG results in a large local immune response by induced expression of cytokines in the urine and bladder tissue. [9] BCG first attaches to the urothelium via fibronectin and integrins and is then internalized by urothelial cells and captured by the initial innate immune response. [17] It is then that antigen presentation and cytokine release results in the upregulation of major histocompatibility complex (MHC) II, IL-6, Il-8 and granulocytemacrophage colony-stimulating factor (GM-CSF). [17] This stimulates local recruitment of immune cells such as granulocytes, CD4 and CD8 T cells, natural killer (NK) cells and macrophages that produce $\mathrm{TH}-1$ cytokines. [17] According to Prescott et al., [18] the antitumor activity of BCG in bladder cancer appears to be a local phenomenon confined to the site of administration. In another study, peripheral blood mononuclear cells (PBMC), urine and serum 
were obtained from patients with superficial carcinoma at various times during the course of BCG instillation, and found an increase in systemic immune activity. [19] Further research is required to determine the precise mechanisms of action of BCG and advance our current knowledge of the immunological processes involved.

\section{Intravesical Treatment-Related Adverse Events and Risk for ARDS}

With knowledge of the recent SARS-CoV-2 pandemic, and the risk for severe complications such as ARDS, it is necessary to review the side effects of intravesical treatment in an attempt to determine its safety in COVID-19 and immunocompromised patients. One major drawback of intravesical BCG is that it is associated with more side effects when compared with intravesical chemotherapy, though more serious adverse outcomes are encountered in less than $5 \%$ of patients. [6] Though still debated, pulmonary complications appear to be of two categories: hypersensitivity reactions and mycobacterial pneumonia. An interstitial pattern on chest radiography, lymphocytosis on bronchial alveolar lavage (BAL) and absence of granulomas on lung biopsy, as well as negative sputum and tissue cultures, are indicative of hypersensitivity response. [20] Mycobacterial pneumonia is characterized by biopsies revealing granulomata and radiographic evidence of consolidation, although testing for acid-fast bacteria (AFB) may be negative. [20] A large analysis of 2602 patients treated with intravesical BCG revealed $0.4 \%$ of patients developed life-threatening BCG sepsis, and $0.7 \%$ developed granulomatous pneumonitis. [21]

With regard to other intravesical therapy, MMC has little systemic absorption due to low molecular weight, and chemical cystitis is common. [22]

\section{Safety of Intravesical BCG in Immunocompromised Patients}

Intravesical BCG is generally considered to be a relative contraindication and used with caution in immunocompromised patients based on current guidelines. However, there have been few studies that have examined the use of intravesical BCG in bladder cancer patients that are concurrently immunosuppressed. In addition to concerns of disseminated infection in immunocompromised patients, BCG is dependent on an effective immune response to exert its mechanism of action in the case of bladder cancer. This has raised concerns that even if instillation is safe in the immunocompromised population, treatment may be ineffective. Herr and Dalbagni [23] discovered that BCG therapy was safe in immunocompromised patients with high-risk bladder tumours and may achieve similar results to non-immunosuppressed patients. However, the sample size was small, and generalization was cautioned. A retrospective chart review of 24 immunosuppressed patients (e.g. concurrent lymphoma, chronic lymphocytic leukemia or on corticosteroid therapy) receiving intravesical BCG concluded that the side effects profile associated with BCG therapy in these patients was comparable with those in patients with no evidence of immunosuppression. [24] Additionally, a number of studies have demonstrated safe use in transplant patients, $[25,26]$ and in patients with concurrent human immunodeficiency virus (HIV) infection. [27]

Therefore, the use of intravesical BCG may be feasible in patients that are concurrently immunocompromised, but larger trials are required for definitive answers. Though immunosuppressed patients with COVID-19 are not at increased risk for more severe pulmonary disease compared with the general population [16], intravesical BCG should be used on a case by case basis in this population until definitive outcomes are determined with further studies.

\section{Efficacy of Intravesical Treatment in Immunological Disorders}

With the possibility that BCG may exhibit a systemic immune response, future research may be warranted regarding the use of BCG in the fight against viruses such as SARS-CoV-2 and various immunological disorders. There has been promising research in recent years exploring the use of BCG against viruses and other immunological disorders with which to build from and guide future studies. Additionally, new clinical trials are being planned to explore the efficacy of boosting immune responses with BCG in the fight against COVID19. [4]

Recently, a study published in 2019 by Gofrit et al. [28] followed 1371 patients for 1 year after their diagnosis with bladder cancer. During follow-up, 65 patients developed Alzheimer's disease (AD), a condition in which the immune system is a major contributor to pathogenesis. Of the patients who developed AD, 21 (2.4\%) were treated with intravesical $\mathrm{BCG}$, while 44 (8.9\%) did not receive BCG therapy. It was determined that patients treated with intravesical BCG manifested more than 4-fold less risk for $\mathrm{AD}$ than those not treated with intravesical BCG.

In the field of virology, BCG vaccination was shown to protect against a non-related viral infection in an experimental model of human yellow fever virus, and it was discovered that the IL-1 pathway is essential for an efficient induction of trained immunity in humans, which may have important implications for vaccination and the pathogenesis of autoinflammatory diseases. [29] Finally, BCG vaccination impacts the immune response to subsequent infections, resulting in reduced morbidity and mortality, and may protect against various DNA and RNA viruses, including herpes and influenza viruses. [30] In these unprecedented times of the SARS- 
CoV-2 pandemic, BCG may offer the potential to boost the immune system in the critical early stages of COVID-19.

\section{Conclusion}

The recent and ongoing SARS-CoV-2 pandemic is a rapidly evolving situation, with many questions remaining unanswered. The pathophysiology of COVID-19-related ARDS and death appears to be heavily related to a cytokine storm resulting in inflammatory damage of host lung tissue. Strategies to boost the immune system in the early stages of the disease may help in preventing it from progressing to a more severe respiratory stage.

Compared with other intravesical therapies, BCG appears to have a higher risk of severe respiratory complications such as ARDS, and its concurrent use in patients with COVID-19 should be carefully monitored. Additionally, these side effects are rare and preventable with proper administration. Although there is no current strong evidence, intravesical BCG may be boosting the immune system and may be playing a role towards decreasing morbidity and mortality of COVID-19. Following disease control, multicentric studies should aim to retrospectively look for the correlation between intravesical BCG and development of COVID-19.

Authors' Contribution Cale Leeson: literature review, manuscript writing Asmaa Ismail: literature review

Mohamed Hashad: literature review and manuscript review

Hazem Elmansy: manuscript revision

Walid Shahrour: manuscript revision

Owen Prowse: manuscript revision

Ahmed Kotb: literature review, manuscript writing and revision

\section{Compliance with Ethical Standards}

Conflict of Interest The authors declare that they have no conflicts of interest.

\section{References}

1. Sahin AR, Erdogan A, Agaoglu PM, Dineri Y, Cakirci AY, Senel ME, et al. 2019 novel coronavirus (COVID-19) outbreak: a review of the current literature. EJMO. 2020;4(1):1-7.

2. World Health Organization. Coronavirus disease 2019 (COVID19): situation report, 75.

3. He F, Deng Y, Li W. Coronavirus disease 2019 (COVID-19): what we know? J Med Virol. 2020;92(7):719-725.

4. de Vrieze J. Can a century-old TB vaccine steel the immune system against the new coronavirus [Internet]? American Association for the Advancement of Science. 2020 [cited 2020 Apr 7]. Available from: https://www.sciencemag.org/news/2020/03/can-century-oldtb-vaccine-steel-immune-system-against-new-coronavirus\#.

5. KassoufW, Traboulsi SL, Kulkarni GS, Breau RH, Zlotta A, Fairey $\mathrm{A}$, et al. CUA guidelines on the management of non-muscle invasive bladder cancer. Can Urol Assoc J. 2015;9(9-10):E690.
6. Chang SS, Boorjian SA, Chou R, Clark PE, Daneshmand S, Konety BR, et al. Diagnosis and treatment of non-muscle invasive bladder cancer: AUA/SUO guideline. J Urol. 2016;196(4):1021-9.

7. Babjuk M, Böhle A, Burger M, Capoun O, Cohen D, Compérat $\mathrm{EM}$, et al. EAU guidelines on non-muscle-invasive urothelial carcinoma of the bladder: update 2016. Eur Urol. 2017;71(3):447-61.

8. Chou R, Selph S, Buckley DI, Fu R, Griffin JC, Grusing S, et al. Intravesical therapy for the treatment of nonmuscle invasive bladder cancer: a systematic review and meta-analysis. J Urol. 2017;197(5):1189-99.

9. BÖHLE A, Brandau S. Immune mechanisms in bacillus CalmetteGuerin immunotherapy for superficial bladder cancer. J Urol. 2003;170(3):964-9.

10. Shi Y, Wang Y, Shao C, Huang J, Gan J, Huang X, Bucci E, Piacentini M, Ippolito G, Melino G. COVID-19 infection: the perspectives on immune responses.

11. Prompetchara E, Ketloy C, Palaga T. Immune responses in COVID-19 and potential vaccines: lessons learned from SARS and MERS epidemic. Asian Pac J Allergy Immunol. 2020;38:1-9.

12. Huang C, Wang Y, Li X, Ren L, Zhao J, Hu Y, et al. Clinical features of patients infected with 2019 novel coronavirus in Wuhan, China. Lancet. 2020;395(10223):497-506.

13. Wu C, Chen X, Cai Y, Zhou X, Xu S, Huang H, et al. Risk factors associated with acute respiratory distress syndrome and death in patients with coronavirus disease 2019 pneumonia in Wuhan, China. JAMA Intern Med. 2020;180(7):1-11.

14. Guan WJ, Ni ZY, Hu Y, Liang WH, Ou CQ, He JX, et al. Clinical characteristics of coronavirus disease 2019 in China. N Engl J Med. 2020;382(18):1708-20

15. Cascella M, Rajnik M, Cuomo A, Dulebohn SC, Di Napoli R. Features, evaluation and treatment coronavirus (COVID-19). InStatPearls [Internet] 2020. StatPearls Publishing.

16. D'Antiga L. Coronaviruses and immunosuppressed patients. The facts during the third epidemic. Liver Transplantation. 2020.

17. Alhunaidi O, Zlotta AR. The use of intravesical BCG in urothelial carcinoma of the bladder. ecancermedicalscience. 2019;13.

18. Prescott S, Jackson AM, Hawkyard SJ, Alexandroff AB, James K. Mechanisms of action of intravesical bacille Calmette-Guerin: local immune mechanisms. Clin Infect Dis. 2000;31(Supplement 3): S91-3.

19. Taniguchi K, Koga S, Nishikido M, Yamashita S, Sakuragi T, Kanetake H, et al. Systemic immune response after intravesical instillation of bacille Calmette-Guerin (BCG) for superficial bladder cancer. Clin Exp Immunol. 1999;115(1):131-5.

20. Rosati Y, Fabiani A, Taccari T, Ranaldi R, Mammana G, Tubaldi A. Intravesical BCG therapy as cause of miliary pulmonary tuberculosis. Urol J. 2016;83(1):49-53.

21. Lamm DL, Van Der Meijden AP, Morales A, Brosman SA, Catalona WJ, Herr HW, et al. Incidence and treatment of complications of bacillus Calmette-Guerin intravesical therapy in superficial bladder cancer. J Urol. 1992;147(3 Part 1):596-600.

22. Koya MP, Simon MA, Soloway MS. Complications of intravesical therapy for urothelial cancer of the bladder. J Urol. 2006;175(6): 2004-10.

23. Herr HW, Dalbagni G. Intravesical bacille $\mathrm{C}$ almette-G uérin $(\mathrm{BCG})$ in immunologically compromised patients with bladder cancer. BJU Int. 2013;111(6):984-7.

24. Yossepowitch O, Eggener SE, Bochner BH, Donat SM, Herr HW, Dalbagni G. Safety and efficacy of intravesical bacillus CalmetteGuerin instillations in steroid treated and immunocompromised patients. J Urol. 2006;176(2):482-5.

25. Palou J, Angerri O, Segarra J, Caparrós J, Guirado L, Diaz JM, et al. Intravesical bacillus calmette-gueĖrin for the treatment of superficial bladder cancer in renal transplant patients. Transplantation. 2003;76(10):1514-6. 
26. Tomaszewski JJ, Larson JA, Smaldone MC, Hayn MH, Jackman SV. Management of bladder cancer following solid organ transplantation. Adv Urol. 2011;2011:1-7.

27. Gaughan EM, Dezube BJ, Bower M, Aboulafia DM, Bohac G, Cooley TP, et al. HIV-associated bladder cancer: a case series evaluating difficulties in diagnosis and management. BMC Urol. 2009;9(1):10.

28. Gofrit ON, Klein BY, Cohen IR, Ben-Hur T, Greenblatt CL, Bercovier H. Bacillus Calmette-Guérin (BCG) therapy lowers the incidence of Alzheimer's disease in bladder cancer patients. PLoS One. 2019;14(11):e224433.
29. Arts RJ, Moorlag SJ, Novakovic B, Li Y, Wang SY, Oosting M, et al. BCG vaccination protects against experimental viral infection in humans through the induction of cytokines associated with trained immunity. Cell Host Microbe. 2018;23(1):89-100.

30. Moorlag SJ, Arts RJ, van Crevel R, Netea MG. Non-specific effects of BCG vaccine on viral infections. Clin Microbiol Infect. 2019;25(12):1473-8.

Publisher's Note Springer Nature remains neutral with regard to jurisdictional claims in published maps and institutional affiliations. 